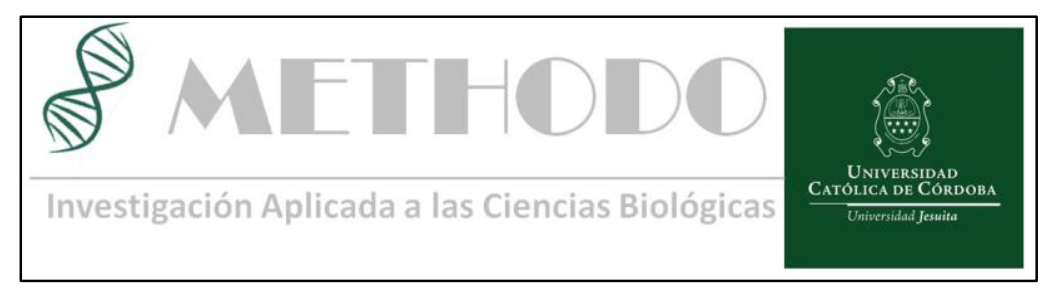

BIOESTADISTICA Y METODOLOGIA APLICADA Methodo 2018 Mar;3(2):38-42 DOI: $10.22529 / \mathrm{me} .2018 .3(2) 05$

Recibido 30 Nov 2017 | Aceptado 03 Ene 2018 | Publicado 30 Jun 2018

\title{
El uso de broncodilatadores en la bronquiolitis
}

\section{The use of bronchodilators in bronchiolitis}

\author{
Alegre $\mathrm{G}^{1}$, Alvares $\mathrm{R}^{2}$, Kreiker $\mathrm{N}^{2}$, Palmero L ${ }^{2}$, Herrera Andino $\mathrm{ML}^{2}$, Cuestas $\mathrm{E}^{3}$.
}

\begin{abstract}
Resumen
Introducción: La bronquiolitis es una patología frecuente en la práctica clínica pediátrica. Constituye una de las principales entidades clínicas dentro de las infecciones respiratorias agudas bajas (IRAB). Se define como el primer episodio de sibilancias con manifestaciones clínicas de infección viral en un niño menor de dos años y afecta principalmente a lactantes menores de 6 meses. El tratamiento es fundamentalmente de soporte con oxigenoterapia e hidratación, quedando el resto de las terapéuticas bajo una profunda controversia acerca de su real efectividad.

Metodología: Desde la exposición de un escenario clínico se planteó una pregunta estructurada para luego realizar una búsqueda bibliográfica con el fin de dar respuesta a la pregunta sobre la efectividad en el tratamiento del bronquiolitis con broncodilatadores. Se realizó una búsqueda en PubMed utilizando los Mesh (Medical Subject Headings) "bronchodilators AND bronchiolitis", se aplicaron distintos filtros, tras lo cual se llegó al número final de 23 artículos. Tras una lectura de los títulos y resúmenes de los artículos, se escogió el artículo "Bronchodilators for bronchiolitis".

Análisis del artículo: Nos hemos centrado las siguientes variables con el fin de responder a la pregunta clínica: la mejoría en el score clínico, la reducción de la tasa de internación y la saturometría de oxígeno. Realizamos luego un análisis secundario de los datos de las distintas variables con el fin de definir los valores de riesgo relativo (RR), reducción de riesgo absoluto (RRA), reducción del riesgo relativo (RRR) y el número necesario a tratar (NNT) con sus respectivos intervalos de confianza del 95\% (IC 95\%). También se llevó a cabo un análisis estadístico para evaluar la variable de saturación de oxígeno.

Recomendación final: No está recomendado el uso de broncodilatadores en el manejo del bronquiolitis, ya que no mejoran ninguna de las variables analizadas. Los broncodilatadores no sólo no tienen eficacia, sino que suponen un gasto y presentan efectos adversos indeseables.
\end{abstract}

Palabras claves: bronquiolitis, broncodilatadores, estadística, eficacia, infancia.

\begin{abstract}
Introduction: Bronchiolitis is a frequent pathology in pediatric clinical practice. It constitutes one of the main clinical entities within acute lower respiratory infections. It is defined as the first episode of wheezing with clinical manifestations of viral infection in a child younger than two years and mainly affects infants under 6 months. The treatment is fundamentally support with oxygen therapy and hydration, leaving the rest of the therapeutic under a deep controversy about its real effectiveness.

Methodology: From the exposition of a clinical scenario, a structured question was presented for a bibliographic search in order to answer the question about the effectiveness in the treatment of bronchiolitis with bronchodilators. A PubMed search was performed using the Mesh (Medical Subject Headings) "Bronchodilators AND Bronchiolitis", different filters were
\end{abstract}


applied, and we reached the final number of 23 articles. After a reading of the titles and summaries of the articles, the article "Bronchodilators for Bronchiolitis" was chosen.

Analysis of the article: We have focused the following variables in order to answer the clinical question: the improvement in the clinical score, the reduction of the hospitalization rate and oxygen saturation. Then, we performed a secondary analysis of the data of the different variables in order to define the values of relative risk, absolute risk reduction, relative risk reduction, and the number needed to treat, with their respective 95\% Confidence Intervals. We also carried out the statistical analysis needed to evaluate the oxygen saturation.

Recommendation: The use of bronchodilators in the management of bronchiolitis is not recommended because none of the analyzed variables improve. Bronchodilators not only do not have efficacy, but they suppose an expense and present undesirable adverse effects.

Keywords: Bronchiolitis, Bronchodilators, Statistics, Efficacy, Childhood.

1. Docente Universidad Católica de Córdoba, Argentina, Facultad de Ciencias de la Salud, Cátedra de Inmunología. Clínica Universitaria Reina Fabiola, Servicio de Alergia e Inmunología.

2. Estudiantes de la Práctica Final Obligatoria. Universidad Católica de Córdoba, Argentina,

Facultad de Ciencias de la Salud, Carrera de Medicina.

3. Profesor Titular. Universidad Católica de Córdoba, Argentina, Facultad de Ciencias de la Salud, Cátedra de Bioestadística.

\section{Introducción}

\section{Escenario Clínico}

Niño de 4 meses de edad sin antecedentes patológicos previos es traído a la consulta por tos y dificultad respiratoria de 24 hs de evolución. Al examen físico se encuentra con tos seca, taquipnea, tiraje subcostal e irritabilidad. Los padres refieren que los días previos a este episodio el niño había presentado un cuadro de vía aérea superior con rinorrea, tos y fiebre de 2 días de evolución. El médico pediatra que lo asiste le diagnostica bronquiolitis y le indica tratamiento ambulatorio con salbutamol en aerosol, lavados nasales y antitérmicos. El paciente inicialmente mejora la sintomatología, pero luego los síntomas recurrieron, por lo que sus padres realizan una interconsulta con otro profesional de la salud. Luego del interrogatorio y del examen físico completo, el profesional interviniente reafirma el diagnóstico de bronquiolitis y se pregunta sobre la función de los broncodilatadores en la disminución de la tasa de internación, la mejora en el score clínico y la optimización de la saturación de oxígeno en el tratamiento del bronquiolitis en niños lactantes.

\section{Pregunta clínica estructurada}

¿En niños lactantes con bronquiolitis (pacientes) son eficaces los broncodilatadores (intervención) para disminuir la posibilidad de internación, mejorar la saturación de oxígeno, y optimizar el score clínico (resultado)?

\section{Contexto clínico ${ }^{1-2}$}

La bronquiolitis se define como el primer episodio de sibilancias con manifestaciones clínicas de infección viral en un niño menor de dos años. Constituye una de las principales entidades clínicas de las infecciones respiratorias agudas bajas. Afecta con mayor frecuencia a lactantes, principalmente a aquellos menores de 6 meses. Se suelen presentar en otoño e invierno.

El agente etiológico más frecuente es el virus sincicial respiratorio (VRS) responsable del 60\%$80 \%$ de los casos de bronquiolitis. Así mismo, se han identificado con menor frecuencia otros agentes virales como, por ejemplo: el rinovirus, para influenza, metapneumovirus, influenza, adenovirus, enterovirus humano y mycoplasma. La infección viral da lugar a la inflamación difusa y aguda de las vías aéreas inferiores, que genera la obstrucción de la vía aérea pequeña.

El cuadro clínico está caracterizado por síntomas de infección respiratoria alta de 1 a 3 días previos (rinorrea, tos $\mathrm{y}$, eventualmente, fiebre de escasa magnitud), seguido de síntomas de obstrucción bronquial periférica (tos, taquipnea, retracción intercostal, espiración prolongada, crepitantes finos, sibilancias, reales) que pueden durar 5 o 6

Revista Methodo: Investigación Aplicada a las Ciencias Biológicas. Facultad de Ciencias de la Salud. Universidad Católica de Córdoba. Jacinto Ríos 571 Bo Gral. Paz. X5004FXS. Córdoba. Argentina. Tel.: (54) 3514517299 / Correo: methodo@ucc.edu.ar / Web: methodo.ucc.edu.ar | BIOESTADISTICA Y METODOLOGIA APLICADA Methodo 2018 $\operatorname{Mar} ; 3(2): 38-42$ 
días. También puede haber síntomas sistémicos como diarrea y vómitos. La recuperación clínica completa se obtiene pasadas dos o tres semanas (tiempo de regeneración del epitelio ciliado). El diagnóstico de bronquiolitis es clínico por lo que no son necesarios exámenes complementarios de rutina.

En cuanto al tratamiento, podemos decir que es fundamentalmente de soporte, con oxigenoterapia e hidratación. El oxígeno es el único medicamento con probada eficacia en bronquiolitis ya que es broncodilatador, vasodilatador y disminuye el trabajo respiratorio. El tratamiento de la obstrucción bronquial es controversial. Los corticoides no están indicados ya que no existe evidencia de que tengan un efecto beneficioso. Lo mismo sucede con la solución salina hipertónica. Otros tratamientos que no se recomiendan: los anticolinérgicos, mucolíticos, antibióticos, antileucotrienos y solución fisiológica nebulizada ya que tampoco han demostraron efectos beneficiosos.

Por lo general los pacientes con bronquiolitis sin factores de riesgo, pueden ser tratados de forma ambulatoria y evolucionan de manera favorable sin complicaciones. En menor medida pueden desarrollar complicaciones tales como apnea, deshidratación, insuficiencia respiratoria aguda / paro cardiorrespiratorio (muerte) y escape aéreo (neumotórax, neumomediastino, enfisema subcutáneo). A largo plazo, atelectasias persistentes, sibilancias recurrentes y enfermedad pulmonar crónica pos infecciosa (EPCPI).

\section{Estrategia de búsqueda}

Para dar respuesta a la pregunta clínica estructurada, se realizó una búsqueda en PubMed, utilizando los MeSH (Medical Subject Headings) "bronchodilators AND bronchiolitis". El resultado obtenido inicialmente fue de 710 artículos, tras lo cual se aplicaron los filtros en relación al tipo de artículo buscado revisiones sistemáticas, con respecto a la edad que sean en población pediátrica y teniendo en cuenta los artículos de los últimos 5 años. Así fue que se obtuvo un total de 23 artículos referentes al tema. Tras una lectura de los títulos y resúmenes de los artículos, se seleccionó el artículo "Bronchodilators for bronchiolitis"3, el cual presenta un nivel de evidencia $1 \mathrm{a}^{4}$

\section{Resumen del artículo}

Los autores que realizaron esta revisión tuvieron como objetivo evaluar los efectos de los broncodilatadores en resultados clínicos en niños de 0 a 12 meses de edad con bronquiolitis aguda. La pregunta inicial que los autores se formularon fue acerca de que, si los broncodilatadores son realmente mejores que el placebo en el manejo del bronquiolitis en niños, medida como mejoramiento de la saturación de oxígeno, del score clínicos, el número de internaciones, la duración de las mismas, pruebas de función pulmonar o el tiempo de resolución de la enfermedad.

Revisaron las evidencias sobre el efecto de los broncodilatadores en los niños con bronquiolitis realizando una búsqueda en CENTRAL 2013, Issue 12, MEDLINE (1996 hasta la segunda semana de enero de 2014) y EMBASE (1998 a enero de 2014). En cuanto a los criterios de selección, buscaron ensayos clínicos aleatorizados controlados, que comparan la efectividad de los broncodilatadores con placebo en el tratamiento del bronquiolitis.

Dentro del estudio se incluyeron treinta ensayos, con un total de 1992 niños con bronquiolitis. Además, analizaron por separado los estudios realizados en pacientes ambulatorios $y$ en pacientes hospitalizados. Todos los broncodilatadores se incluyeron en la revisión, excepto la epinefrina porque ésta se encuentra analizada en otra revisión de Cochrane. El albuterol (también conocido como salbutamol) se utiliza comúnmente en los estudios, por lo que también se revisó este broncodilatador como un subgrupo.

Como resultados obtuvieron que en once estudios de pacientes internados y en diez de pacientes no hospitalizados, la saturación de oxígeno no mejoraría con el uso de broncodilatadores. Por otro lado, en los pacientes ambulatorios el tratamiento broncodilatador no disminuyó la tasa de hospitalización, y en los pacientes internados el uso de broncodilatadores no redujo el tiempo de hospitalización. Con respecto a la oximetría los efectos estimados para los pacientes internados fueron ligeramente mayores que en los pacientes ambulatorios. Los resultados de la oximetría revelaron una significativa heterogeneidad. En ocho de los estudios de pacientes internados que fueron analizados no se observó ningún cambio en el promedio del score clínico tras el tratamiento broncodilatador. En nueve estudios de pacientes ambulatorios que fueron internados el promedio del score clínico disminuyó ligeramente con el empleo de broncodilatadores. Un hallazgo estadísticamente significativo, pero con una importancia clínica cuestionable. El score clínico reveló una heterogeneidad significativa. Incluyendo sólo los estudios con bajo riesgo de sesgo se redujo la heterogeneidad, pero tuvo un

Revista Methodo: Investigación Aplicada a las Ciencias Biológicas. Facultad de Ciencias de la Salud. Universidad Católica de Córdoba. Jacinto Ríos 571 Bo Gral. Paz. X5004FXS. Córdoba. Argentina. Tel.: (54) 3514517299 / Correo: methodo@ucc.edu.ar / Web: methodo.ucc.edu.ar | BIOESTADISTICA Y METODOLOGIA APLICADA Methodo 2018 Mar;3(2):38-42 
pequeño impacto en el puntaje clínico médico general o en los tratamientos dentro del ámbito hospitalario. Dentro de los efectos adversos de los broncodilatadores observados se incluyen taquicardia, disminución de la saturación de oxígeno y temblor.

Los autores concluyeron que los broncodilatadores no incrementan la saturación de oxígeno, no reducen el tiempo de hospitalización, ni disminuyen el tiempo de resolución de la enfermedad en pacientes tratados de forma ambulatoria y tampoco reduce la tasa de internación en pacientes ambulatorios. Debido a los efectos adversos y al gasto asociados a los broncodilatadores, estos no son útiles en el manejo de rutina del bronquiolitis.

Sin embargo, finalizan admitiendo que el estudio se encuentra limitado por el pequeño tamaño de la muestra y la falta de un diseño estandarizado y de resultados validados en todos los estudios. Sostienen que se necesitan futuros ensayos con muestras mayores, con una metodología estandarizada y métodos de asesoramiento consistentes para responder a la pregunta de la eficacia de los broncodilatadores en el tratamiento del bronquiolitis.

\section{Análisis del artículo}

Para el análisis del artículo nos hemos centrado en tres variables principales a estudiar con el fin de responder a la pregunta clínica, que son: la mejoría en el score clínico, la reducción de la tasa de internación y la saturometría de oxígeno. Para ello se realizó un análisis secundario de los datos de las distintas variables con el fin de definir los valores de riesgo relativo (RR), reducción de riesgo absoluto (RRA), reducción del riesgo relativo (RRR) y el número necesario a tratar (NNT) con sus respectivos intervalos de confianza del $95 \%$ (IC 95\%). Con estos datos obtuvimos información sobre la mejoría en el score clínico y la reducción en la tasa de internación. Para la variable de la saturación de oxígeno nos centramos en los valores de las medias de la saturación de oxígeno obtenido en los diferentes trabajos de investigación y se realizó el análisis estadístico correspondiente para definir si había diferencia significativa entre los diferentes grupos.

A continuación, se describen los resultados obtenidos con respecto a la mejoría en el score clínico y con respecto a la reducción de la tasa de internación teniendo en cuenta los siguientes datos: de los 80 pacientes bajo tratamiento con broncodilatadores un $47,5 \%$ presentaron mejoría en el score clínico y del grupo de pacientes tratados con placebo un $84,4 \%$ mejoraron su score clínico.
Además, se tuvo en cuenta que la prevalencia de internaciones de los pacientes tratados con broncodilatadores, de un total de 222, fue del $16,6 \%$ y la de los pacientes bajo tratamiento con placebo, de un total de 182 pacientes, fue un $23,6 \%$.

Analizando la RRA para ambas variables, los resultados fueron los siguientes: para la mejoría del score clínico de un $37 \%$ a favor del placebo con un IC $95 \%$ de 0,233 a 0,505; y para la reducción de la tasa de internación fue de un $7 \%$ a favor de la utilización de los broncodilatadores, con un IC $95 \%$ de $-0,010$ a 0,148 . En ambos casos se puede definir que ninguna de las dos variables mejora con el uso de broncodilatadores en comparación con el uso de placebo.

Con respecto a la RRR fue del $43,7 \%$ (IC $95 \%$ de $28-60 \%$ ) en la mejoría del score clínico con la utilización del placebo. En tanto que el valor obtenido en la reducción de la tasa de internación fue de $29,6 \%$ con un IC $95 \%$ de $-4 \%$ a $63 \%$. Siendo no beneficioso el úso de broncodilatadores.

Luego de analizar los valores del NNT, para la mejoría del score clínico el NNT fue de 3 (IC 95\% de 2 a 4) a favor del uso del placebo y para la reducción de la tasa de internación fue de 14 (IC 95\% de 7 a 102), también a favor del uso de placebo. Por lo que sigue sin ser efectivo el uso de broncodilatadores en esta patología.

Al realizar el análisis estadístico de la diferencia de las medias con respecto a la saturación de oxígeno no se obtuvo una diferencia significativa con respecto al uso de broncodilatadores o de placebo $(\mathrm{p}=0,8892)$.

\section{Comentarios y recomendaciones finales}

Hoy en día se cuestiona si el uso de broncodilatadores es eficiente $\mathrm{o}$ no en ésta patología. Debido a ello, decidimos realizar la búsqueda de evidencias publicadas, lo cual nos puede orientar a definir mejor el manejo y las acciones a realizar en estos casos, y si realmente se justifica el uso de broncodilatadores debido a sus efectos adversos y el gasto que ello supone.

Como se ha comentado previamente, hicimos un análisis estadístico de un meta análisis que revisa este tema de manera estadísticamente confiable. De allí estudiamos las variables del score clínico, la tasa de internación y la saturación de oxígeno, para poder comparar el efecto de los broncodilatadores frente al placebo y así llegar a una respuesta más confiable para nuestro interrogatorio.

Luego del análisis pudimos concluir que no está recomendado el uso de dichos medicamentos en el

Revista Methodo: Investigación Aplicada a las Ciencias Biológicas. Facultad de Ciencias de la Salud. Universidad Católica de Córdoba. Jacinto Ríos 571 Bo Gral. Paz. X5004FXS. Córdoba. Argentina. Tel.: (54) 3514517299 / Correo: methodo@ucc.edu.ar / Web: methodo.ucc.edu.ar | BIOESTADISTICA Y METODOLOGIA APLICADA Methodo 2018 
manejo del bronquiolitis, ya que no mejoran ninguna de las variables analizadas. Podemos afirmar que, con la evidencia disponible no se recomienda el uso de broncodilatadores de manera empírica en pacientes con bronquiolitis como tratamiento ambulatorio. Los broncodilatadores no sólo no tienen eficacia al no reducir el índice de internaciones, ni el score clínico, ni los registros de saturación, sino que suponen un gasto y a su vez tienen efectos adversos.

\section{Bibliografía}

1. Abramovich $\mathrm{N}$, Aguerre $\mathrm{V}$, et all. Recomendaciones para el manejo de las infecciones respiratorias agudas bajas en menores de 2 años. SAP [Internet]; 2015 [consultado 8 sept 2017]. Disponible en http://www.sap.org.ar.

2. Collard Borsotti MV, Panario A. En Gomila AA, et all. Pediatría clínica I y II, lera edición. Córdoba. Editorial de la Universidad Católica de Córdoba; 2012. p 553-555.

3. Gadomski AM, Scribani MB. Bronchodilators for bronchiolitis. Cochrane
Library [Internet] 2014 Jun [consultado 20 agosto 2017]; (6). p1-75. Disponible en http://www.cochranelibrary.com.

4. Asenjo-Lobos C, Manterola C. Jerarquización de la evidencia. Niveles de evidencia y grados de recomendación de uso actual. Rev chilena Infectol. 2014; 31 (6). p 705

Link a la transmisión del ateneo central Facultad Ciencias de la Salud 12-10-2017

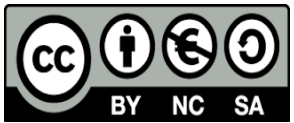

